\title{
PAPER
}

\section{Behavior of adaptive algorithms in active noise control systems with moving noise sources}

\author{
Akira Omoto, Daisuke Morie and Kyoji Fujiwara \\ Kyushu Institute of Design, \\ Shiobaru 4-9-1, Minami-ku, Fukuoka, 815-8540 Japan \\ (Received 15 March 2001, Accepted for publication 5 September 2001)
}

\begin{abstract}
Adaptive algorithms such as LMS are often used in active noise control systems to update the output of secondary sources. If we apply the active scheme to traffic noise control, it is important to understand the behavior of the adaptive algorithm in response to significant changes of the primary noise due to movement of the noise source. This paper describes preliminary investigations of the tracking ability of adaptive algorithms whilst the noise source is moving. The well-known filtered-x LMS, NLMS and RLS algorithms are used in the simulations. In addition, experiments are conducted to verify the results of the simulations.
\end{abstract}

Keywords: Active noise control, Adaptive algorithm, Moving noise source

PACS number: 43.50.Ki

\section{INTRODUCTION}

The authors have applied the active control technique to suppress diffracted sound by a noise barrier [1,2], in which the multiple error filtered-x LMS algorithm [3] was used to examine the effect of the control relative to a stationary noise source. However, if we try to extend our findings to traffic noise control, the assumption of a stationary environment is apparently insufficient due to significant changes of the primary paths.

An important problem is determining how an adaptive algorithm behaves in a system with large changes of the noise signal. There have been several investigations on the behavior of the LMS algorithm in a non-stationary environment [4-7]. In most of these works a system identification problem was used as an example. However, the analysis of a system with primary and secondary acoustic paths, which is common in active noise control, has seldom been investigated.

The authors have reported the results of preliminary investigations involving a moving noise source, in which filtered-x LMS were used as the adaptive algorithm [8].

However, this LMS algorithm is known as a rather slow convergence algorithm. In practical situations involving an active noise control system, it would be interesting to compare performance with other algorithms having faster convergence speed.

Simple free field is assumed, and numerical simulations examined the behavior of well-known algorithms, such as filtered-x LMS and RLS, with moving primary noise sources. Furthermore, experiments were conducted to verify the results of the numerical simulations and to provide realistic guidelines for the design of noise control systems.

\section{ANALYTICAL MODEL}

Figure 1(a) shows the effective block diagram of the active noise control systems of the feedforward structure. First, a single input and single output system, using $U$-th order FIR adaptive filter $\boldsymbol{u}$, is assumed to simplify the problem. Multi-channel case is discussed later. The $d(n)$ is the primary signal, which can be obtained at each sampled time $n$ by convoluting the noise signal $s(n)$ with the time varying impulse response $\boldsymbol{p}(n)$. Thus,

$$
\begin{aligned}
d(n) & =\boldsymbol{p}(n) * s(n) \\
& =\sum_{i=0}^{P-1} p_{i}(n) s(n-i)
\end{aligned}
$$

where

$$
\boldsymbol{p}(n)=\left[p_{0}(n) p_{1}(n) \cdots p_{P-1}(n)\right]
$$

provided that the impulse response can be modeled as $P$-th order FIR filter.

The impulse response from the noise source to the noise detecting sensor, which is modeled as $Q$-th order FIR filter,

$$
\boldsymbol{q}(n)=\left[q_{0}(n) q_{1}(n) \cdots q_{Q-1}(n)\right]
$$




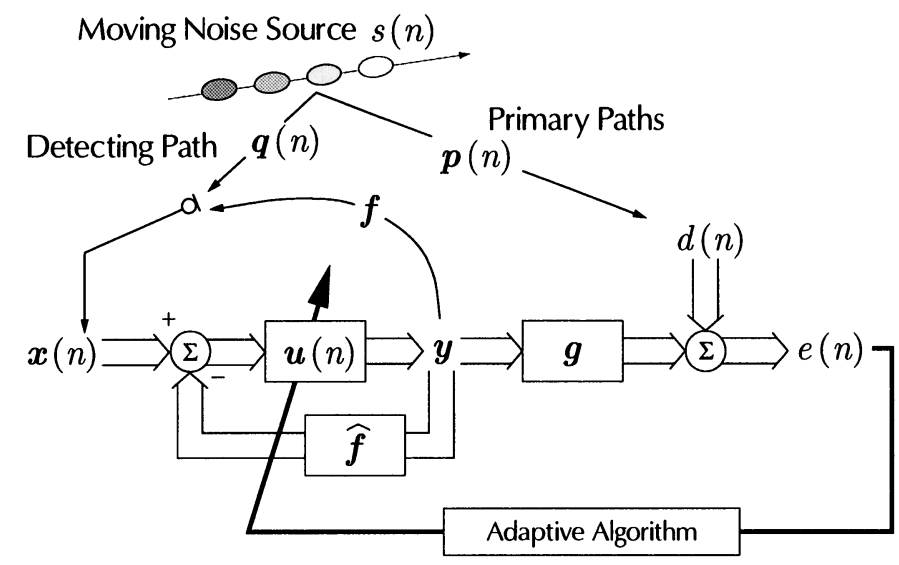

(a) Block diagram of the feedforward system.

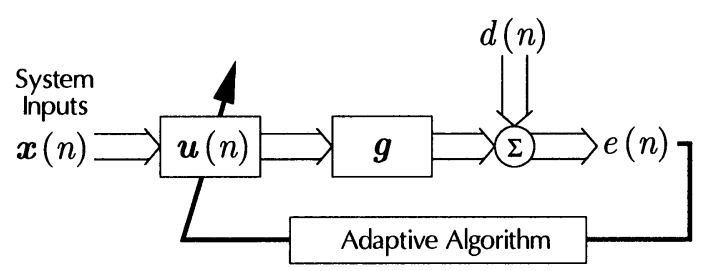

(b) Equivalent block diagram.

Fig. 1 Block diagram of the feedforward active noise control system.

also varies with time due to the movement of the source. The input signal $x(n)$ can be expressed as

$$
\begin{aligned}
x(n) & =\boldsymbol{q}(n) * s(n) \\
& =\sum_{i=0}^{Q-1} q_{i}(n) s(n-i) .
\end{aligned}
$$

The variable $e(n)$ represents the error signal and $g$ is the impulse response between the secondary source and the error sensor which is modeled as $G$-th order filter expressed as

$$
\boldsymbol{g}=\left[g_{0} g_{1} \cdots g_{G-1}\right] .
$$

The $U$-th order adaptive FIR filter $\boldsymbol{u}(n)$ is updated iteratively to minimize the squared output of the error signal. Acoustic feedback $\boldsymbol{f}$ exists from the output of the secondary source to the noise sensor. However, if the feedback canceller is introduced [9] and the model of the acoustic feedback paths $\widehat{\boldsymbol{f}}$ is sufficiently accurate to express the real feedback path $f$, the equivalent block diagram can be simplified as shown in Fig. 1(b), and the system can be expressed as

$$
\begin{aligned}
e(n) & =d(n)+\boldsymbol{g} * \boldsymbol{u}(n) * x(n) \\
& =d(n)+\sum_{i=0}^{G-1} g_{i} \sum_{j=0}^{U-1} u_{j}(n-i) x(n-i-j),
\end{aligned}
$$

where $u_{j}$ means the $j$-th element of the adaptive filter $\boldsymbol{u}$.
Noise signal $s(n)$ in the calculations was the exhaust noise of an idling motorbike, recorded near the rear tire. The sound was $\mathrm{A} / \mathrm{D}$ converted at a sampling frequency of $8 \mathrm{kHz}$. The impulse responses between the source and the sensors were modeled by a simple delay [8]. The distances between source and the sensors determined the position and amplitude of the nonzero element, as shown in Fig. 2.

The position of the nonzero element was determined as Position $=\operatorname{round}\left(R(n) / c \times f_{\mathrm{s}}\right)$, where $R(n)$ is the distance from the primary source to each sensor - which varies with iteration index $n, c$ is the sound speed, and $f_{\mathrm{s}}$ is the sampling frequency. The round function rounds the argument number to the nearest integer.

Figure 3 shows the arrangement of the source and the sensors for the numerical simulation. A noise detection sensor was located at $(x, y)=(4.0,0)[\mathrm{m}]$. The movement of the primary source along the $x=5 \mathrm{~m}$ line was modeled as follows:

1. Standing still at $y=y_{0} \mathrm{~m}$ for initial $T_{\mathrm{s}}$ second.

2. Accelerate along the function of $[\sin (t)+1] / 2$, (t: $-\pi / 2 \sim \pi / 2)$ until $V[\mathrm{~km} / \mathrm{h}]$ during $T_{\mathrm{a}}$ seconds.

3. Moving subsequently at a constant speed of $V[\mathrm{~km} / \mathrm{h}]$.

For comparison, calculations were made on the

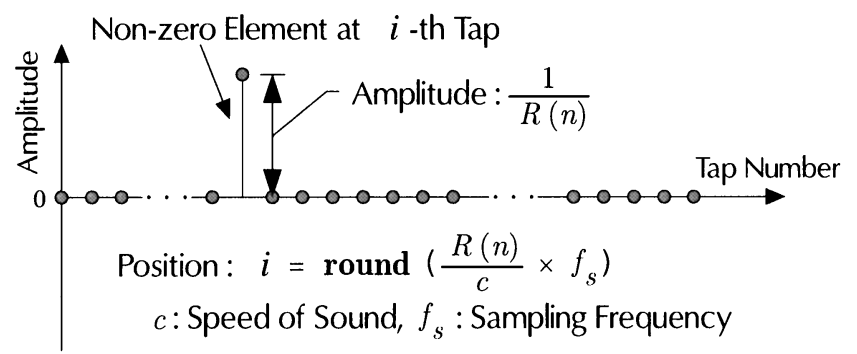

Fig. 2 Impulse response used in the simulations in which $R(n)$ is the distance between each source and sensor, $c$ is the speed of sound, and $f_{\mathrm{s}}$ is a sampling frequency.

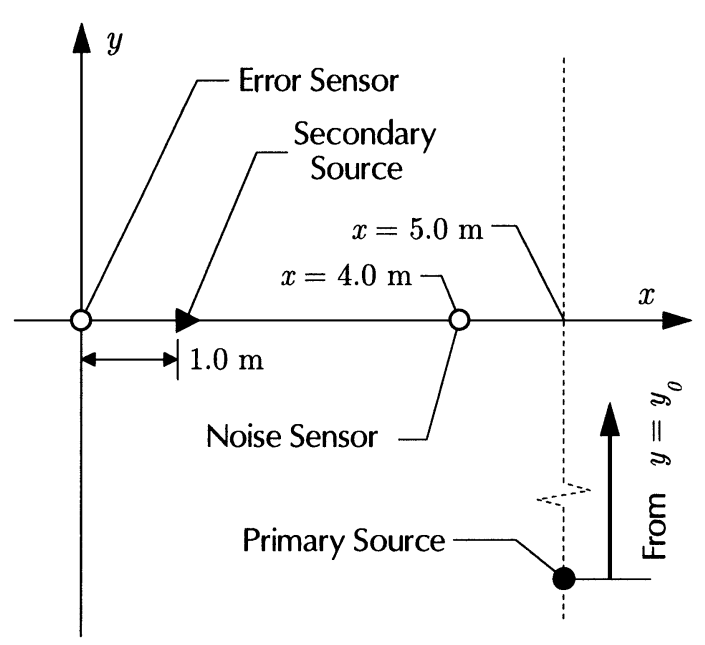

Fig. 3 Arrangement of the primary source, the secondary source, and the error sensor in the free field. 
assumption that the sound source stood still at location $(x, y)=(5,0)[\mathrm{m}]$. The sound source and both sensors were assumed to be point source and point sensors respectively. The algorithms used in the simulations were filtered-x LMS, NLMS, and RLS algorithms. The weight update procedures for these algorithms are listed below:

filtered-x LMS [3]

$$
\boldsymbol{u}(n+1)=\boldsymbol{u}(n)-\mu \boldsymbol{r}(n) e(n)
$$

in which $\mu$ is step size parameter and $\boldsymbol{r}(n)$ is the filtered reference signal defined as

$$
\begin{gathered}
r(n)=\boldsymbol{g} * x(n) \\
\boldsymbol{r}(n)=[r(n) r(n-1) \cdots r(n-U+1)],
\end{gathered}
$$

NLMS [10]

$$
\boldsymbol{u}(n+1)=\boldsymbol{u}(n)-\frac{\mu}{\gamma+U \hat{\boldsymbol{\Phi}}} \boldsymbol{r}(n) e(n)
$$

where $\gamma$ is a small number, $\hat{\Phi}$ is estimated power of the input signal $r(n)$ and is updated as

$$
\hat{\Phi}(n)=(1-\beta) \hat{\Phi}(n-1)+\beta r^{2}(n),
$$

in which $\beta$ is the smoothing parameter,

RLS [10]

$$
\boldsymbol{u}(n+1)=\boldsymbol{u}(n)-\boldsymbol{k}(n) e(n)
$$

where

$$
\begin{aligned}
\boldsymbol{k}(n) & =\frac{\boldsymbol{z}(n)}{\boldsymbol{r}(n) \boldsymbol{z}(n)+1}, \\
\boldsymbol{z}(n) & =\lambda^{-1} \boldsymbol{Q}(n-1) \boldsymbol{r}^{T}(n), \\
\boldsymbol{Q}(n) & =\lambda^{-1} \boldsymbol{Q}(n-1)-\boldsymbol{k}(n) \boldsymbol{z}^{T}(n),
\end{aligned}
$$

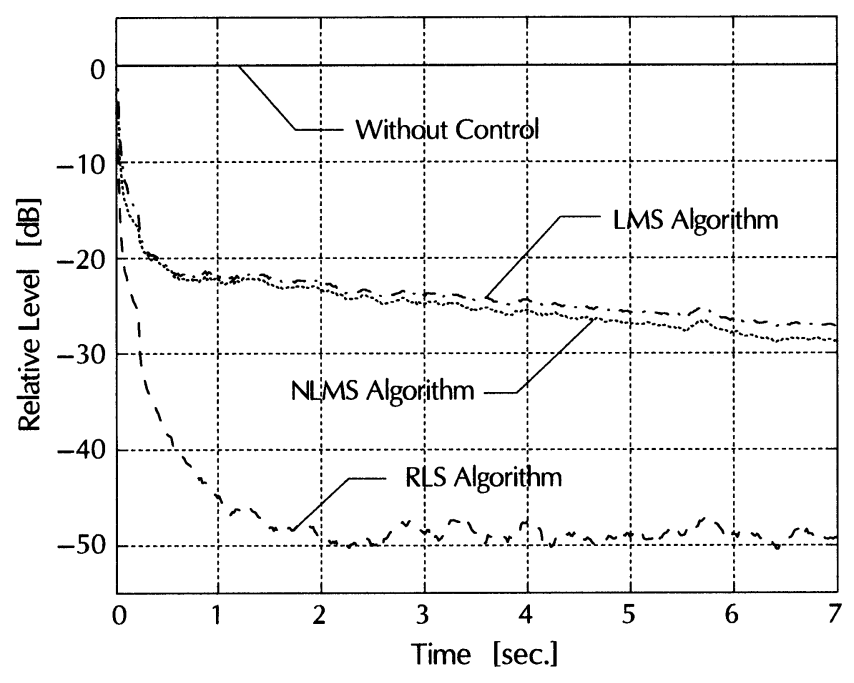

(a) standing noise source. in which $\boldsymbol{k}$ is $U \times 1$ Kalman gain vector, $z$ is $U \times 1$ temporary vector, $\boldsymbol{Q}(U \times U)$ is equivalent to the inversion of the autocorrelation matrix of $x(n)$, and $\lambda$ is the forgetting factor.

In the practical calculation, sampling frequency was $8 \mathrm{kHz}$ and the length of the adaptive filter $U=256$, the lengths of the impulse responses $G=256, P=32,768$, $Q=32,768$ were assumed.

\section{DISCUSSIONS}

\subsection{Single Channel Case}

Figure 4 shows the results when the noise source is standing still (a) and moving (b). In the case of a moving noise source, $y_{0}=-80 \mathrm{~m}, T_{\mathrm{s}}=0.5 \mathrm{~s} T_{\mathrm{a}}=3 \mathrm{~s}$ and the speed of $V=80 \mathrm{~km} / \mathrm{h}$ were assumed. The parameters of $\mu$ and $\lambda$ are important factors which may restrict the speed of convergence and the performance of the system. These values were determined after trial and error.

The curves in the figures indicate the level of the RMS value of the error sensor output, and they are normalized relative to the levels obtained without control.

If the source was standing still, the RLS algorithm displayed the fastest convergence and largest attenuation relative to other algorithms. The LMS and NLMS algorithms showed a similar behavior, albeit with a slower convergence speed (Fig. 4(a)). If the source was moving, however, the LMS and NLMS simulations showed better results than the RLS simulation, with effective attenuation being observed within the calculated time period. On the other hand, the RLS algorithm produced an increase of the output level when the noise source crossed the face of the system.

Since the RLS algorithm uses all of the time history

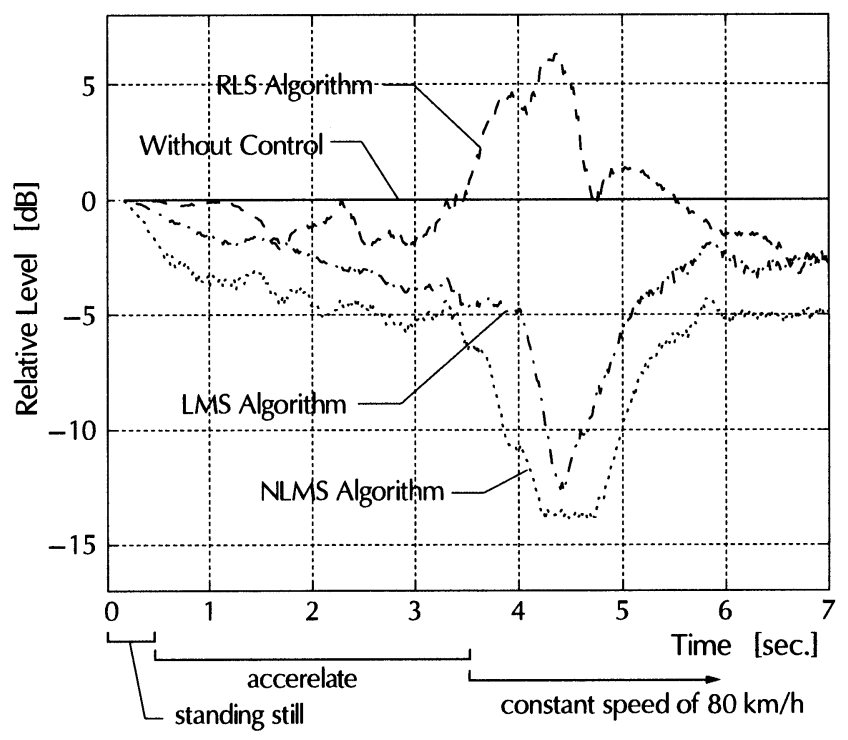

(b) moving noise source.

Fig. 4 Changes in the squared output of error sensor when the primary source is standing still (a) and moving (b). 
data in updating the adaptive filter coefficients, significant changes in the noise signal may have caused the degradation in the performance of the system. This tendency can be observed even in the initial stationary period. Since the initial position of the noise source was $80 \mathrm{~m}$ away from the sensors, no signals reached the control points when the sound source had begun to emit the noise ( $0 \mathrm{~s}$ in the figure). Algorithm recognized the arrival of signals at around $0.2 \mathrm{~s}$. RLS algorithm seemed to be dull for this 'sudden' change. The LMS and NLMS algorithms on the other hand, which only use current data when undergoing coefficient updating, were more effective when noise signal changed significantly.

The results obtained here suggest that the LMS type algorithm may be able to adapt to changes of the primary signal resulting from the movement of the noise source.

\subsection{Multi Channel Case}

Results in the preceding section indicated that the LMS type algorithm would work effectively with a moving noise source. If we extend the system to multi-channel, there will be another practical problem, such as effect of the number of noise detecting sensors. Another simulations are thus performed with multiple error filtered-x LMS (MEFXLMS) algorithm [3].

Four channel system (4-error sensors and 4-secondary sources) is assumed as shown in Fig. 5, and the number of the noise sensors is varied from 1 to 4 channels. The choice of the noise sensors in each calculation are detailed in Fig. 5.

The movement of the noise source is same as the single

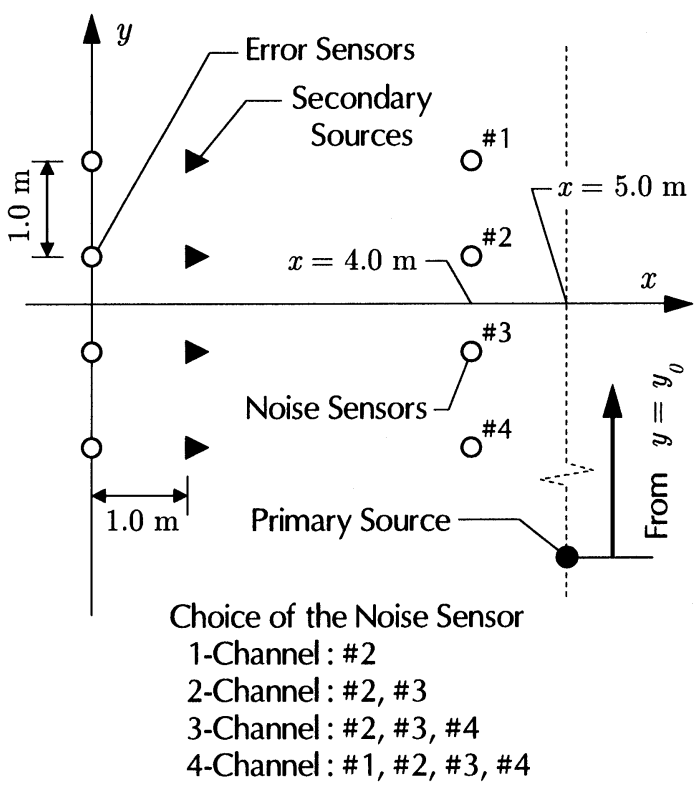

Fig. 5 Arrangement of the primary source, the noise sensors, the secondary sources, and the error sensor in the free field.

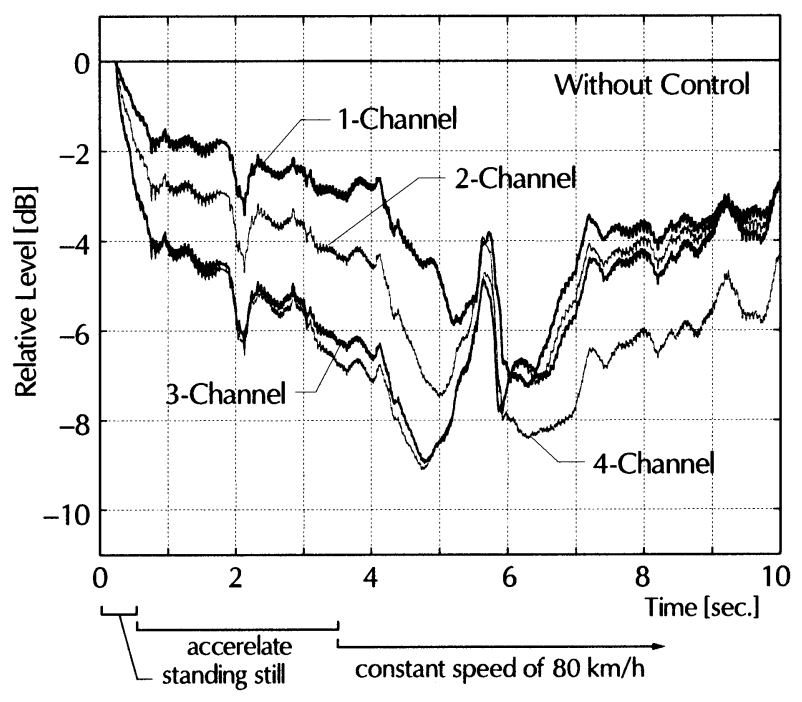

Fig. 6 Changes in the averaged sum of the squared output from the error sensors when the number of the noise sensors was varied.

channel case, i.e., standing still at $y=-80 \mathrm{~m}$ for initial $0.5 \mathrm{~s}$, and accelerate until $80 \mathrm{~km} / \mathrm{h}$ during $3 \mathrm{~s}$ and moving subsequently at constant speed.

Figure 6 shows the results. The curves in the figure indicate the normalized level of the sum of the squared output of the error sensors which are smoothed by the same procedure as RMS with time constant of $0.2 \mathrm{~s}$.

Attenuation of the error signal before $6 \mathrm{~s}$ increased as the number of the noise sensors increased from 1 to 3 channels. However, significant differences were not observed between the cases using 3 and 4 noise sensors. Four channel case showed the advantageous results in the time period of 6 to $10 \mathrm{~s}$. The noise source passed the face of the system at about $5.7 \mathrm{~s}$. The noise sensor \#1 (which was nearest to the noise source after $5.7 \mathrm{~s}$ ) worked effectively after the noise source passed over the system. These results may suggest that a suitable number of noise sensors should be selected in the design of the control systems.

\section{EXPERIMENT}

Experiments in an anechoic chamber were conducted to verify the results of the numerical simulations and to investigate a more realistic experimental design, involving variation in the number of noise sensors and additionally, the choice of sampling frequency used in digital signal processing.

Real time operation DSP hardware used in the experiment (Redec EX-Tool) could select the number of sensors and secondary outputs - both up to 4 channels, and a sampling frequency of up to $48 \mathrm{kHz}$. A system of 4 channels was used for the error sensors and secondary sources. The number of noise sensor varied from 1 to 4 channels. 


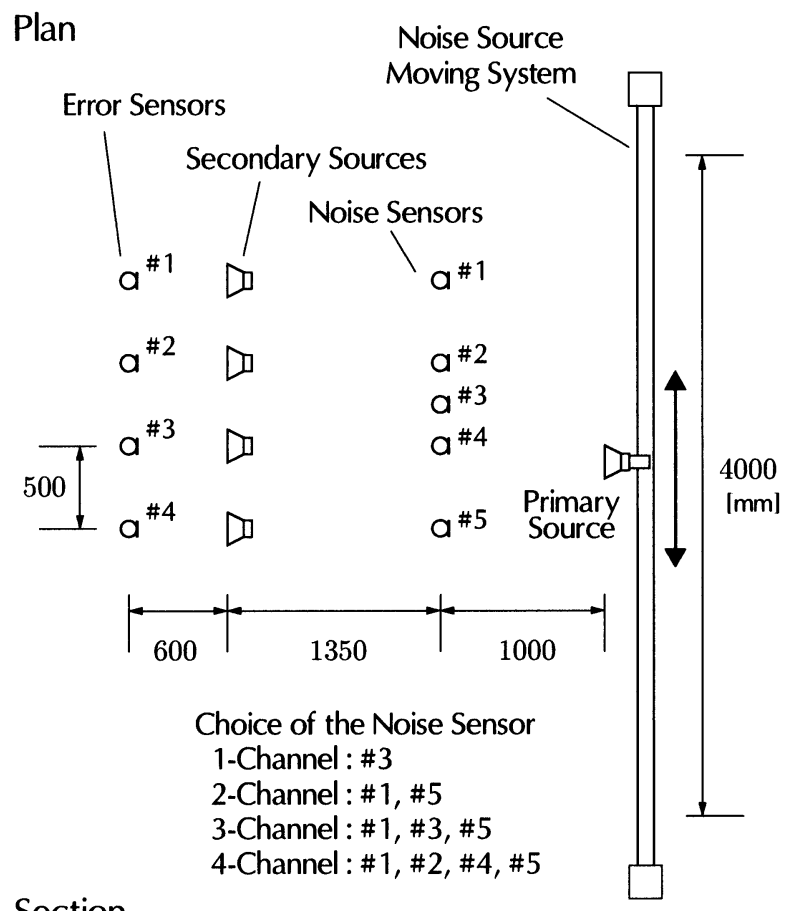

Section

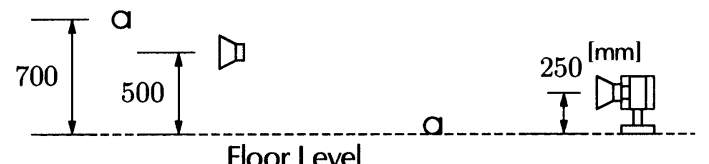

Fig. 7 Arrangement of the sources and the sensors in the anechoic chamber, in which the dimensions are in $\mathrm{mm}$.

Arrangement of sensors and sources is shown in Fig. 7, as are details of specific noise sensors used in each experiment. The sound source moving system had a running extent of 4 meters, allowing reciprocated motion of a loudspeaker which acted as a noise source with an appointed velocity of $1 \mathrm{~m} / \mathrm{s}$. The noise signal was the exhaust noise of a motorbike recorded near the rear tire during driving.

The algorithm used in the experiment was one of the LMS family algorithms - the MEFX-LMS with leakage term. The Error Scanning method [11] was also introduced to reduce calculation. The length of the adaptive filter was assumed to be 256. Computational complexity made it unrealistic to describe the multichannel RLS algorithm in this hardware.

Figure 8 shows the results obtained when the number of noise sensors was varied from 1 to 4 . The sampling frequency was fixed in $12 \mathrm{kHz}$ in this experiment. The curves on the figure indicate the power spectrum of the output signal from the error sensor \#1. These values were obtained after 512 times additive average during the state in which the system seemed to have converged almost.

Attenuation of the output signal below $200 \mathrm{~Hz}$ increased as the number of noise sensors increased from

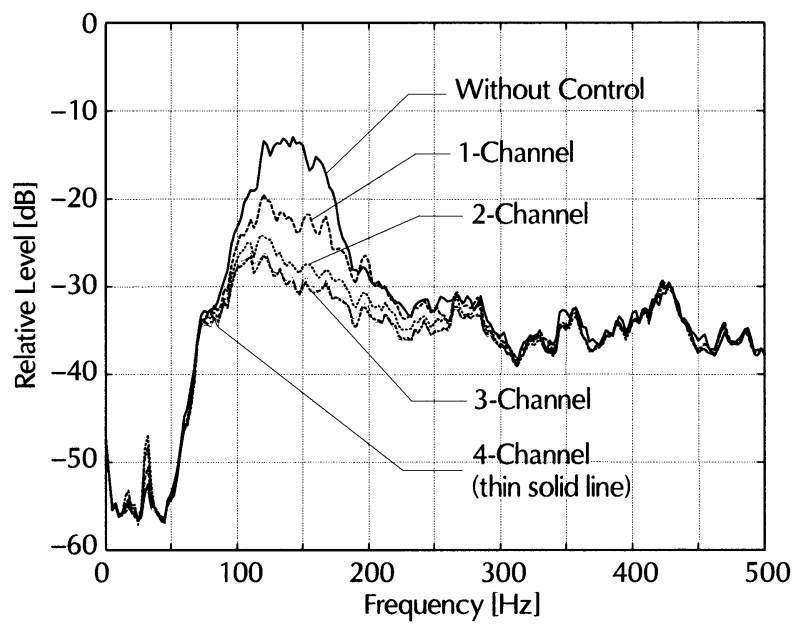

Fig. 8 Changes in the averaged power spectrum of the output signal from error sensor \#1 when the number of the noise sensors was varied.

1 to 3 channels. However, large differences were not observed between experiments using 3 and 4 noise sensors. Similar results were obtained with other error sensors. These results again suggest that a suitable number of noise sensors should be selected in the design of noise control systems. In this experimental arrangement with reciprocating movement of noise source, three noise sensors were enough to obtain the possible attenuation.

Figure 9 shows the results obtained when the sampling frequency was varied, and displays the averaged power spectrum observed at error sensor \#1. In this case, the number of noise sensors was fixed in 4 . As the sampling frequency increased from $6 \mathrm{kHz}$ to $12 \mathrm{kHz}$, the attenuation increased.

An experiment was also conducted in which the primary noise source was moved with a speed of $2 \mathrm{~m} / \mathrm{s}$. The results obtained in this experiment were quite similar

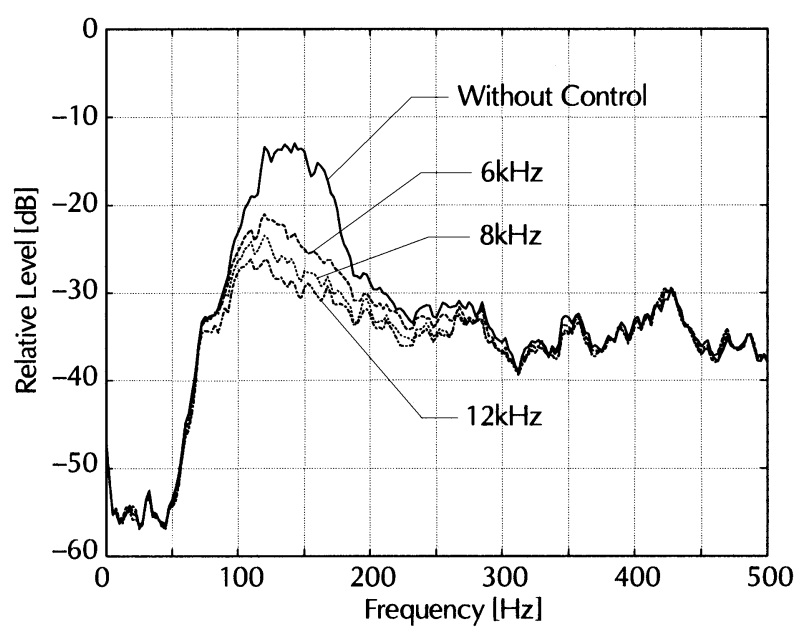

Fig. 9 Changes in the averaged power spectrum of the output signal from error sensor \#1 when the sampling frequency was varied. 
to the results shown in Figs. 8 and 9. Due to the limitation of the moving system in these experiments, the speed of the primary source was rather slower than the speed of more realistic sources of noise. Consequently, a more realistic experimental design is our current focus.

\section{CONCLUDING REMARKS}

The behavior of the well-known adaptive algorithms filtered-x LMS, NLMS, and RLS — has been investigated with respect to a moving primary noise source. Numerical simulations yielded the following results:

- when the sound source is stationary the RLS algorithm converged faster and attenuation was larger, relative to the LMS and NLMS algorithms.

- the performance of the RLS algorithm fell greatly when sound source moved.

- the LMS algorithm showed the best behavior, and significant attenuation was obtained even in the case of a moving source.

The characteristics of the RLS algorithm, which used all the time data to update the coefficients, appeared to be the cause of its poor tracking ability. Furthermore, if minimal computational complexity is considered, it may be said that it is advantageous to use the LMS algorithm in an active control system when the sound source moves.

As Haykin [12] suggested, the speed of convergence of an algorithm and its ability to track changes in a signal are separate considerations. The selection of an algorithm should be done according to individual case.

An experiment was also carried out to verify the effectiveness of the LMS algorithm with real time signal processing hardware. The results showed the importance of the number of noise sensors utilized and the selection of sampling frequency. Attenuation increased as sampling frequency increased. Similarly, attenuation increased when the number of noise sensors increased. However, moderate numbers of noise sensors were shown to exist as viable possibilities. We can conclude for the present study that the best method in dealing with a moving noise source is to select an algorithm from the LMS family whilst utilizing the highest possible sampling frequency.

\section{ACKNOWLEDGMENT}

The authors would like to acknowledge Mr. K. Kurihara and Mr. S. Awata, Redec Co., Ltd. for their helpful assistance in the experimental study. This work was supported by a Grant-in-Aid for Scientific Research (11750524) from Japan Society for the Promotion of Science.

\section{REFERENCES}

[1] A. Omoto and K. Fujiwara, "A study of an actively controlled noise barrier", J. Acoust. Soc. Am., 94, 2173 (1993).
[2] A. Omoto, K. Takashima and K. Fujiwara, "Active suppression of sound diffracted by a barrier: An outdoor experiment", J. Acoust. Soc. Am., 102, 1671 (1997).

[3] S. J. Elliott, I. M. Stothers and P. A. Nelson, "A multiple error algorithm and its application to active control of sound and vibration", IEEE Trans. Acoust. Speech Signal Process., ASSP-35, 1423 (1987).

[4] B. Widrow, J. M. McCool, M. G. Larimore and C. R. Johnson, Jr., "Stationary and nonstationary learning characteristics of the LMS adaptive filter", Proc. IEEE, 64, 1151 (1976).

[5] N. J. Bershad and O. Macchi, "Comparison of RLS and LMS algorithm for tracking a chirped signal”, Proc. ICASSP, 89, 896 (1989).

[6] O. Macchi and N. J. Bershad, "Adaptive recovery of a chirped sinusoid in noise, Part 1: Performance of the RLS algorithm", IEEE Trans. Acoust. Speech Signal Process., ASSP-39, 583 (1991).

[7] N. J. Bershad and O. Macchi, "Adaptive recovery of a chirped sinusoid in noise, Part 2: Performance of the LMS algorithm", IEEE Trans. Acoust. Speech Signal Process., ASSP-39, 595 (1991).

[8] A. Omoto, T. Matsui and K. Fujiwara, "The behaviour of an adaptive algorithm with a moving primary source", J. Acoust. Soc. Jpn. (E), 19, 211 (1998).

[9] P. A. Nelson and S. J. Elliott, Active Control of Sound (Academic Press, London, 1992).

[10] S. M. Kuo and D. R. Morgan, Active Noise Control Systems (John Wiley \& Sons, Inc., New York, 1996).

[11] H. Hamada, "Signal processing for active control", Proc. 1991 Acoust. Soc. Jpn. Int. Symp. on Active Control of Sound and Vibration, p. 33 (1991).

[12] S. Haykin, Adaptive Filter Theory, 2nd ed. (Prentice Hall, Englewood Cliffs, 1991).

Akira Omoto graduated from the Kyushu Institute of Design, Fukuoka, Japan, in 1987, and received the Dr. Eng. degree from the University of Tokyo, Japan, in 1995, for a dissertation on the active noise barrier. From 1987 to 1991, he worked as a Research and Development Engineer at Nittobo Acoustic Engineering Co., Ltd., Tokyo. In 1991, he was appointed Research Assiciate at the Department of Acoustic Design, Kyushu Institute of Design, and was made Associate Professor in 1997. His current research interests include active noise control and the sound field design in recording studios.

Daisuke Morie was born in 1977. He received B. Design. and M. Design degrees from Kyushu Institute of Design in 1999 and 2001, respectively. He is currently working as a visiting researcher at Mechanical Engineering Department, California State University, Long Beach. His research interests include active noise control and adaptive algorithm.

Kyoji Fujiwara was born in Hyogo on August 11, 1946. He graduated from Kobe University in 1969, and received M. E. degree from Kobe University in 1971. He received Dr. Eng. degree from the University of Tokyo in 1979. He began to work as a Research Associate at the Department of Acoustic Design, Kyushu Institute of Design from 1971, and was promoted to Associate Professor in 1985. Since 1989, he is Full Professor and working on the research field of noise barrier, absorbing structure and sound field analysis. He is now a member of Acoust. Soc. Jpn., Acoust Soc. Am, Architectural Inst. of Jpn, and INCE/Japan. 